Please share your stories about how Open Access to this article benefits you.

\title{
Calculation of the interfacial free energy of a binary hard-sphere fluid at a planar hard wall
}

\author{
by Jesse L. Kern and Brian B. Laird
} 2013

This is the published version of the article, made available with the permission of the publisher. The original published version can be found at the link below.

Kern, Laird. (2013). Calculation of the interfacial free energy of a binary hard-sphere fluid at a planar hard wall. Journal of Chemical Physics 140(24703):24703

Published version: http://www.dx.doi.org/10.1063/1.4858433

Terms of Use: http://www2.ku.edu/ scholar/docs/license.shtml 


\section{AIP $\mid$ The Jounal of Chemical Physics}

\section{Calculation of the interfacial free energy of a binary hard-sphere fluid at a planar hard}

wall

Jesse L. Kern and Brian B. Laird

Citation: The Journal of Chemical Physics 140, 024703 (2014); doi: 10.1063/1.4858433

View online: http://dx.doi.org/10.1063/1.4858433

View Table of Contents: http://scitation.aip.org/content/aip/journal/jcp/140/2?ver=pdfcov

Published by the AIP Publishing

\section{Articles you may be interested in}

The anisotropic hard-sphere crystal-melt interfacial free energy from fluctuations

J. Chem. Phys. 125, 094710 (2006); 10.1063/1.2338303

Solid-liquid interfacial free energy of small colloidal hard-sphere crystals

J. Chem. Phys. 119, 7467 (2003); 10.1063/1.1607307

The solid-liquid interfacial free energy of close-packed metals: Hard-spheres and the Turnbull coefficient

J. Chem. Phys. 115, 2887 (2001); 10.1063/1.1391481

Toward determining the true hardsphere interfacial free energy

J. Chem. Phys. 102, 8283 (1995); 10.1063/1.468961

On the structure of binary hardsphere fluid mixtures

J. Chem. Phys. 77, 4227 (1982); 10.1063/1.444333

\section{AlP Re-register for Table of Content Alerts}




\title{
Calculation of the interfacial free energy of a binary hard-sphere fluid at a planar hard wall
}

\author{
Jesse L. Kern and Brian B. Laird ${ }^{\text {a) }}$ \\ Department of Chemistry, University of Kansas, Lawrence, Kansas 66045, USA
}

(Received 4 November 2013; accepted 12 December 2013; published online 8 January 2014)

\begin{abstract}
Using molecular-dynamics simulation and Gibbs-Cahn Integration, we calculate the interfacial free energy $\gamma$ of a binary hard-sphere fluid mixture at a structureless, planar hard wall. The calculation is performed as a function of packing fraction (density) for several values of the diameter ratio $\alpha$ $=\sigma_{2} / \sigma_{1}$, where $\sigma_{1}$ and $\sigma_{2}$ are the diameters of the two particle types in the mixture. Our results are compared to those obtained from the bulk version of the White Bear Mark II (WBII) classical density-functional theory, which is a modification of the Fundamental-Measure Theory of Rosenfeld. The WBII bulk theory is shown to be in very good agreement with the simulation results, with significant deviation only at the very highest packing fractions. (C) 2014 AIP Publishing LLC. [http://dx.doi.org/10.1063/1.4858433]
\end{abstract}

\section{INTRODUCTION}

The interfacial free energy $\gamma$ is the primary property governing the thermodynamics of solid-liquid interfaces. For chemically heterogeneous interfaces, in which the solid and liquid have a large difference in composition, an important reference system is the hard-sphere (HS) fluid confined at a structureless hard wall (HW). The simple, but non-trivial, nature of such systems is well suited for the evaluation and development of theories for inhomogeneous fluids, especially integral-equation methods or classical density-functional theories (cDFT). In addition, some real polymer and colloid systems can be modeled quantitatively as hard-spheres. For example, it has recently been shown that poly- $N$-isopropylacrylamide (PNIPAM) spheres in a solvent with appropriate charge screening reproduce results from previous calculations on the pure HS system. ${ }^{1}$ There has been considerable theoretical and computational work focused on the HS/HW system; however, the vast majority of such studies has focused on single component HS fluids, with significantly less work examining confined multi-component HS fluids. In this work, we examine the interfacial thermodynamics of a binary hard-sphere fluid at a structureless hard wall using molecular-dynamics simulation.

Although theoretical estimates from Scaled Particle Theory have been in existence since the $1960 \mathrm{~s},{ }^{2,3}$ the first simulations to calculate $\gamma$ for a single component $\mathrm{HS} / \mathrm{HW}$ system were those of Henderson and van Swol, ${ }^{4}$ who obtained $\gamma$ by integrating the excess surface stress obtained from molecular-dynamics simulation. Subsequently, a number of higher precision calculations were reported using thermodynamic integration (TI) ${ }^{5-7}$ Mechanical methods ${ }^{8}$ can also be used, but require considerable computational effort in order to achieve acceptable precision. Laird and Davidchack used the cleaving-wall method ${ }^{9}$ and Gibbs-Cahn Integration ${ }^{10,11}$ to obtain very high precision results for this system that are

\footnotetext{
a) Author to whom correspondence should be addressed. Electronic mail: blaird@ku.edu
}

useful in evaluating theoretical predictions, such as that from $\mathrm{SPT}^{2}$ and recent cDFT results from Fundamental Measure Theory (FMT) ${ }^{12}$ and its extensions. ${ }^{13,14}$

There have been a number of cDFT studies of the structure and interfacial thermodynamics of the binary HS/hard wall system.; ${ }^{13-17}$ however, simulation studies have been limited to evaluations of structure through the calculation of density profiles. ${ }^{18,19}$ This has made it difficult to quantitatively evaluate the thermodynamic predictions of the theoretical studies. In this work, we present results from molecular-dynamics simulation for the interfacial thermodynamics of a variety of binary hard-sphere fluids at a structureless hard wall. In these simulations, the interfacial free energy $\gamma$ is determined using an extension of the Gibbs-Cahn Integration $^{10,11,20-22}$ technique to a multicomponent fluid at a static, structureless solid wall. The results are compared to recent results based on the White Bear II cDFT. ${ }^{14,17}$

\section{SYSTEM}

We consider a binary mixture of hard-sphere particles of differing sizes at a structureless wall. The interparticle and wall-particle potentials $\phi$ and $\phi_{w}$ are, respectively,

$$
\begin{gathered}
\phi(r)=\left\{\begin{array}{ll}
\infty & r<\sigma_{i j} \\
0 & r \geq \sigma_{i j}
\end{array},\right. \\
\text { and } \phi_{w}(z)=\left\{\begin{array}{ll}
\infty & z<\sigma_{i} / 2 \\
0 & z \geq \sigma_{i} / 2
\end{array},\right.
\end{gathered}
$$

where $\sigma_{i}$ is the diameter of spheres of type $i \in\{1,2\}, \sigma_{i j}$ is the arithmetic mean of $\sigma_{i}$ and $\sigma_{j}, r$ the distance between the centers of two spheres, and $z$ the distance between sphere center and wall. Defined in this manner, the sphere-wall collision occurs at the surface of the sphere, not the center.

Our system consists of $N_{1}$ type 1 spheres with diameter $\sigma_{1}$ and $N_{2}$ type 2 spheres with diameter $\sigma_{2}$ in a volume $V$, 
giving a total number density $\rho$ of

$$
\rho=\frac{N_{1}+N_{2}}{V}=\rho_{1}+\rho_{2},
$$

where $\rho_{i}$ is the number density of species $i$. Without loss of generality, we assume $\sigma_{1}>\sigma_{2}$. The mole fraction of each species is $x_{i}=\rho_{i} / \rho$, with $x_{1}+x_{2}=1$. The total packing fraction $\eta$ for the mixture is $\eta=\eta_{1}+\eta_{2}$, where $\eta_{i}=\frac{\pi}{6} \sigma_{i}^{3} \rho_{i}$. For a given $\alpha$, the system is completely described by specifying $x_{1}$ and $\eta$.

\section{GIBBS-CAHN INTEGRATION}

In this work, we calculate the interfacial free energy from MD simulations using the Gibbs-Cahn Integration method, which is based on Cahn's extension ${ }^{23}$ of the surface thermodynamics of Gibbs. ${ }^{24}$ This method has been applied to the calculation of $\gamma$ for one- and two-component crystalmelt interfaces ${ }^{20-22}$ and for single-component fluids at static walls, ${ }^{10,11}$ where it was shown to have considerably less computational overhead than competing methods without sacrificing precision. The derivation of the Gibbs-Cahn adsorption equation specifically for a fluid at a static wall was discussed in Ref. 10 and is outlined here. Consider an $r$-component fluid at a static surface. The total Gibbs free energy $G$ for this system is

$$
G=E-T S+P V,
$$

where $E, T, S$, and $P$ are the internal energy, temperature, entropy, and pressure, respectively. The Gibbs free energy of the bulk fluid $G^{\mathrm{f}}$ is

$$
G^{\mathrm{f}}=\sum_{i=1}^{r} \mu_{i} N_{i}
$$

where $\mu_{i}$ is the chemical potential of species $i$ and superscript "f" denotes bulk fluid quantities. The interfacial free energy is defined as the difference per unit area between the free energy of the fluid at the interface and the bulk fluid.

$$
\gamma A=G-G^{\mathrm{f}}=E-T S+P V-\sum_{i=1}^{r} \mu_{i} N_{i},
$$

where $A$ is the interfacial area. The differential of this quantity is

$$
d(\gamma A)=-S d T+V d P-\sum_{i=1}^{r} N_{i} d \mu_{i} .
$$

This equation and the Gibbs-Duhem equation for the bulk fluid

$$
-S^{\mathrm{f}} d T+V^{\mathrm{f}} d P-\sum_{i=1}^{r} N_{i}^{\mathrm{f}} d \mu_{i}=0
$$

then form a system of two simultaneous linear equations connecting the differentials. Cramer's rule from linear algebra can then be used to eliminate one of the differential terms on the right-hand side of Eq. (7), giving

$$
A d \gamma=-[S / X] d T+[V / X] d P-\sum_{i=1}^{r}\left[N_{i} / X\right] d \mu_{i},
$$

where $X$ can be any variable conjugate to a differential $d x$ in Eq. (9) and the notation $[Y / X]$ is defined as

$$
[Y / X] \equiv \frac{1}{X^{\mathrm{f}}}\left|\begin{array}{ll}
Y & X \\
Y^{\mathrm{f}} & X^{\mathrm{f}}
\end{array}\right| .
$$

In Eq. (10), the quantities with superscript "f" are measured in a region of the system far enough away from the interface to be considered bulk fluid, whereas the unscripted quantities are measured in a region that wholly encompasses the interface and associated interfacial fluid. The form of Eqs. (9) and (10) ensures that one of the $[Y / X] d x$ terms in Eq. (9) can be eliminated by setting $X=Y$.

For a hard-sphere system, such as the one studied here, the temperature dependence of $\gamma$ is a trivial linear scaling, ${ }^{25}$ so the $d T$ term in Eq. (9) can be neglected. Thus, for a binary (2-component) hard-sphere fluid at a hard wall, the GibbsCahn adsorption equation (Eq. (9)) becomes

$$
A d \gamma=[V / X] d P-\left[N_{1} / X\right] d \mu_{1}-\left[N_{2} / X\right] d \mu_{2} .
$$

The application of Eq. (11) requires a specific choice for $X$, which is equivalent to defining a Gibbs Dividing Surface $(\mathrm{GDS})^{26}$ such that the excess surface value of the quantity $X$ is zero. For example, one possible choice is $X=N_{1}$ yielding

$$
\begin{aligned}
d \gamma & =\frac{1}{A}\left[V / N_{1}\right] d P-\frac{1}{A}\left[N_{2} / N_{1}\right] d \mu_{2} \\
& =v_{N_{1}} d P-\Gamma_{2 \mid N_{1}} d \mu_{2},
\end{aligned}
$$

where $v_{N_{1}}$ and $\Gamma_{2 \mid N_{1}}$ are the excess volume and excess number of type 2 particles per unit volume using a GDS defined such that the surface excess number of type 1 particles is zero. Similarly, one can choose $X=N_{2}$ or $V$; however, for this study, we found it most convenient to choose $X$ to be the total particle number, $N=N_{1}+N_{2}$. To do this, we must rewrite Eq. (7) for the binary hard-sphere system as

$$
\begin{aligned}
A d \gamma & =V d P-N_{1} d \mu_{1}-N_{2} d \mu_{2} \\
& =V d P-N_{1} d\left(\mu_{1}-\mu_{2}\right)-N d \mu_{2} \\
& =V d P-N_{1} d \Delta \mu-N d \mu_{2},
\end{aligned}
$$

where $\Delta \mu=\mu_{1}-\mu_{2}$. Equation (11) then becomes

$$
A d \gamma=[V / X] d P-\left[N_{1} / X\right] d \Delta \mu-[N / X] d \mu_{2} .
$$

Setting $X=N$ then gives

$$
d \gamma=v_{N} d P-\Gamma_{1 \mid N} d \Delta \mu
$$

where $v_{N}$ and $\Gamma_{1 \mid N}$ are the excess $V$ and excess $N_{1}$ for a GDS defined such that the excess total number of particles is zero. The choice of $X=N$ was used in the current work because we find it gives significantly smaller statistical error than the other possible choices for similar computational effort and the value of $\Delta \mu$ can be calculated easily using a particle swap method, as described later in this section.

We calculate $\gamma$ by integrating Eq. (15) with respect to pressure at fixed $x_{1}$, recognizing that $\gamma(P=0)=0$. The resulting expression can be written as

$$
\gamma\left(P ; x_{1}\right)=\int_{0}^{P}\left[v_{N}\left(P^{\prime} ; x_{1}\right)-\Gamma_{1 \mid N}\left(\frac{d \Delta \mu}{d P^{\prime}}\right)_{x_{1}}\right] d P^{\prime} .
$$


In this work, we evaluate this integral numerically at fixed $x_{1}$ as a function of $\eta$ for the system, as identity and number of particles are far easier to control than pressure and $\Delta \mu$. We use reduced units throughout with energy and length scales of $k_{B} T$ and $\sigma_{1}$, respectively, giving the following reduced quantities: $\rho_{i}^{*}=\rho_{i} \sigma_{1}^{3}, \gamma^{*}=\gamma \sigma_{1}^{2} / k_{B} T, P^{*}$ $=P \sigma_{1}^{3} / k_{B} T, v_{N}^{*}=v_{N} / \sigma_{1}, \Gamma_{1 \mid N}^{*}=\Gamma_{1 \mid N} \sigma^{2}$, and $\mu_{i}^{*}=\mu_{i} /$ $k_{B} T$. For simplicity, in what follows, the "*” superscript is omitted and all quantities are assumed to be in reduced units, unless otherwise specified.

\section{SIMULATION DETAILS}

Molecular dynamics (MD) simulations are performed on a HS mixture between two structureless hard walls in a slit-pore geometry. The MD simulations were implemented using the linked-cell-list algorithm of Rapaport. ${ }^{27}$ The size of the simulation box is fixed at approximately $L_{x} \times L_{y} \times L_{z}$ $=33 \sigma_{1} \times 33 \sigma_{1} \times 38 \sigma_{1}$ for most systems. At larger values of $\eta$ and smaller values of $x_{1}$, we use a smaller system size of $22 \sigma_{1} \times 22 \sigma_{1} \times 26 \sigma_{1}$ to keep $N<50000$ and computation times reasonable. The $z$-axis is defined as being normal to the walls, and periodic boundary conditions are employed in the $x$ and $y$ directions. The distance between the walls $\left(L_{z}\right)$ is chosen to be large enough that the walls do not interact and there is a significant region of bulk fluid in the center of the simulation. For this initial study diameter ratios of $\alpha=0.7,0.8$, and 0.9 were chosen, as the phase diagrams in this range of $\alpha$ are well characterized. ${ }^{28-30}$

Each system equilibrates for $1000 \tau$, where $\tau$ $=\sigma_{1} \sqrt{m / k_{B} T}$ is the natural time unit for hard-sphere dynamics. Equilibrium averages of $P, \Delta \mu$, and the individual density profiles, $\rho_{1}(z)$ and $\rho_{2}(z)$, are collected for each system studied over a total time of $20000 \tau$, divided into 20 blocks of $1000 \tau$ each. Bulk values such as density and mole fraction are determined by averaging over the bulk region. We found that defining the bulk region to consist of all particles more than $7 \sigma_{1}$ away from either wall was sufficient for all systems studied. Pressures are calculated using the equation

$$
\beta P=\rho^{\mathrm{f}}+\frac{\beta m}{3 V^{\mathrm{f}} \Delta \tau}\left\langle\sum \vec{r}_{i j} \cdot \vec{v}_{i j}\right\rangle,
$$

where $\beta=1 / k_{B} T, V^{\mathrm{f}}$ is the volume of the bulk region, $\Delta \tau$ is the time over which collisions are summed in the bulk fluid, $\vec{r}_{i j}$ is the vector between the centers of two spheres upon collision, $m$ is the mass of the particles and $\vec{v}_{i j}$ is the difference of the velocities of two spheres upon collision. Fig. 1 shows an example of the density profiles calculated for $\alpha=0.9$ at a reduced pressure of 1.6627 and a mole fraction $x_{1}=0.25$.

The chemical potential difference $(\Delta \mu)$ is calculated in a manner similar to the Widom insertion method, ${ }^{31}$ in which individual chemical potentials $\left(\mu_{i}\right)$ are calculated by inserting test particles into the system. To calculate $\Delta \mu$ the Widom insertion method is modified by replacing particle insertions with particle swaps. ${ }^{32,33}$ The advantage of the particle swap method is that it determines $\Delta \mu$ directly, without the need to separately calculate the individual $\mu_{i}$, which is difficult at large $\eta$. Dividing $\Delta \mu$ into an ideal-solution component and

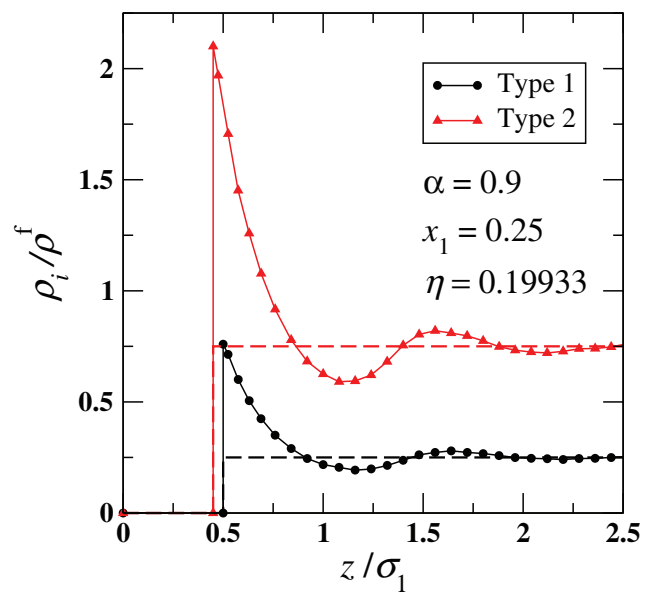

FIG. 1. Sample density profiles near the wall for $\alpha=0.9, x_{1}=0.25$, and $P=1.6627$. The dashed lines represent the density profiles for this $\alpha$ in the zero-pressure limit.

an excess component, we have

$$
\Delta \mu=\Delta \mu_{\text {ideal }}+\Delta \mu_{\text {excess }},
$$

where

$$
\Delta \mu_{\text {ideal }}=\ln \left(\frac{x_{1}}{1-x_{1}}\right),
$$

and, for the hard-sphere binary system, the excess component is given by

$$
\Delta \mu_{\text {excess }}=\ln \left[\frac{\mathcal{P}\left(\sigma_{2 \leftarrow 1}\right)}{\mathcal{P}\left(\sigma_{1 \leftarrow 2}\right)}\right],
$$

where $\mathcal{P}\left(\sigma_{j \leftarrow i}\right)$ is the probability that changing the identity of a randomly chosen particle from type $i$ to type $j$ will not result in a particle overlap. [Note: for the hard-sphere mixtures considered here, $\mathcal{P}\left(\sigma_{2 \leftarrow 1}\right)$ is identically 1.]

The values of $v_{N}$ and $\Gamma_{1 \mid N}$ as functions of pressure (or $\eta$ ) are calculated by numerically integrating their representations as integrals over the density profiles:

$$
\begin{gathered}
v_{N}(P)=\int_{0}^{\infty}\left[1-\frac{\rho(z ; P)}{\rho^{\mathrm{f}}(P)}\right] d z, \\
\Gamma_{1 \mid N}(P)=\int_{0}^{\infty}\left[\rho_{1}(z, P)-x_{1}^{\mathrm{f}}(P) \rho(z, P)\right] d z .
\end{gathered}
$$

Because the density profiles approach step functions in the low density (pressure) limit, the values of $v_{N}(P=0)$ and $\Gamma_{1 \mid N}(P=0)$ can be determined analytically to be $\left[x_{1}+\alpha(1\right.$ $\left.\left.-x_{1}\right)\right] / 2$ and 0 , respectively. Examples of the density profiles in the zero-pressure limit are shown in Fig. 1.

\section{RESULTS AND DISCUSSION}

For each of the diameter ratios studied here $(\alpha=0.7,0.8$, and 0.9 ), we examine the system at mole fractions $x_{1}=0.25$, 0.50 , and 0.75 , each for packing fractions in the range $0<\eta$ $<0.5$. This range of $\eta$ was chosen because it corresponds approximately to the fluid region of the phase diagrams, ${ }^{28-30}$ in order to avoid the freezing transition. Because of differential 


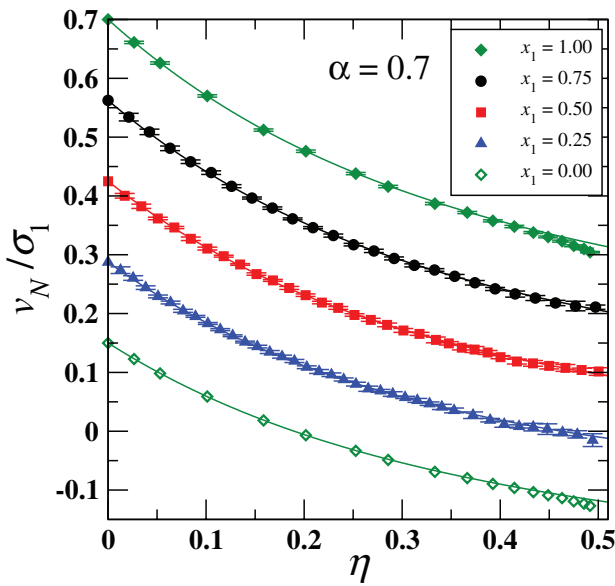

FIG. 2. Excess volume per unit area, $v_{N}$, as a function of packing fraction for the $\alpha=0.7$ mixtures. Results for the pure system (original and scaled) from Laird and Davidchack ${ }^{10}$ are included (diamond symbols). For clarity, the $x_{1}=1.00$ data has been shifted by +0.2 on the $v_{N}$-axis, the $x_{1}=0.75$ data by +0.1 , the $x_{1}=0.25$ data by -0.1 , and the $x_{1}=0.00$ data by -0.2 . The solid lines are the predictions from the WBII bulk theory.

adsorption of one species over the other at the interface, the mole fraction, and density of the bulk fluid region can change slightly from that of the initial configuration as the system equilibrates. Before taking averages, we add (or swap) particles of the requisite type to ensure that the relative deviations from the desired bulk values are less than $10^{-4}$ in the final equilibrated system.

The observed pressures in the simulation are in good agreement with pressure values obtained using both the Mansoori-Carnahan-Starling-Leland equation of state ${ }^{34}$ or the Carnahan-Starling Mark III equation of state ${ }^{14}$ for HS mixtures, as are the calculated chemical potential differences. For more detail, see the supplementary material. ${ }^{35}$

Figs. 2-4 show the results for the excess volume, $v_{N}$, for $\alpha=0.7,0.8$, and 0.9 , respectively. For comparison, we also show in Figs. 2-4 the excess volume as determined from the White Bear II (WBII) bulk theory. ${ }^{35}$ For low and intermediate packing fractions, the WBII expressions are in excellent

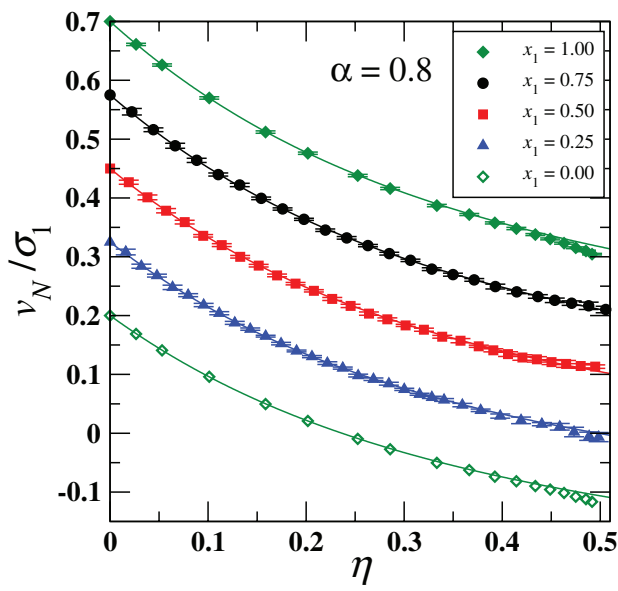

FIG. 3. Excess volume per unit area, $v_{N}$, as a function of packing fraction for the $\alpha=0.8$ mixtures. Same conventions as Fig. 2 .

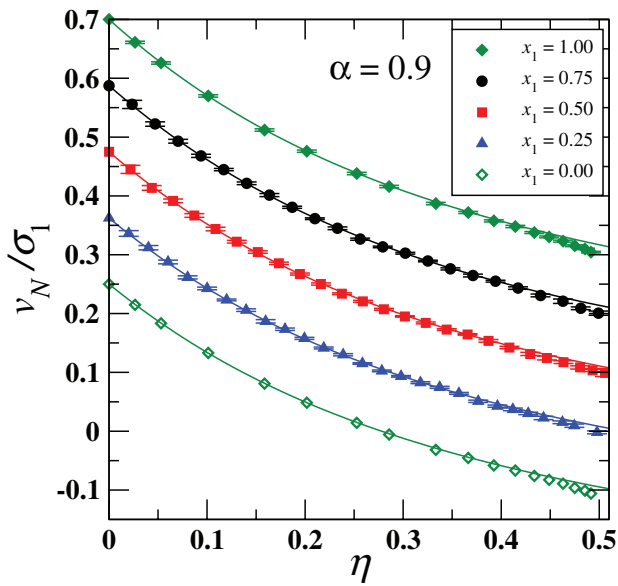

FIG. 4. Excess volume per unit area, $v_{N}$, as a function of packing fraction for the $\alpha=0.9$ mixtures. Same conventions as Fig. 2.

agreement with the simulation results, although some deviation is seen at the highest packing fractions $(\eta>0.45)$. Interestingly, the deviations are seen primarily in the pure systems ( $x_{1}=0$ or 1$)$ and are less pronounced in the mixtures. The data for $v_{N}$ at for all systems at all mole fractions and packing fractions studied are given in tabular form in the supplementary material. ${ }^{35}$

The excess adsorption $\Gamma_{1 \mid N}$ is a measure of segregation of the mixture at the interface. Because $\Gamma_{1 \mid N}$ for these systems is relatively small, it is difficult to get high relative precision for this quantity in reasonable simulation time. This is especially true at the higher packing fractions where the calculation of the $\Gamma_{1 \mid N}$ involves numerical integration over highly oscillatory density profiles. The results for $\Gamma_{1 \mid N}$ for $\alpha=0.7,0.8$, and 0.9 are shown in Figs. 5-7, respectively. These results are also given in tabular form in the supplementary material. ${ }^{35}$ Also shown in Figs. 5-7 are the corresponding predictions from WBII. Except at low packing fractions, the scatter in the data is large - due to the sampling issues discussed above; however, the contribution of $\Gamma_{1 \mid N}$ to the value of $\gamma$ through the integration of Eq. (16) is also quite small, so the scatter in

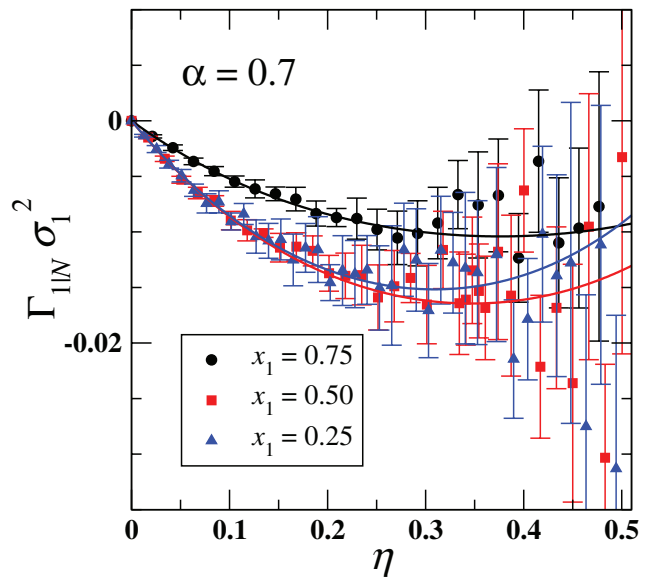

FIG. 5. Interfacial excess particle number $\Gamma_{1 \mid N}$ as a function of packing fraction for the $\alpha=0.7$ systems. The corresponding predictions from the WBII bulk theory are shown as solid lines. 


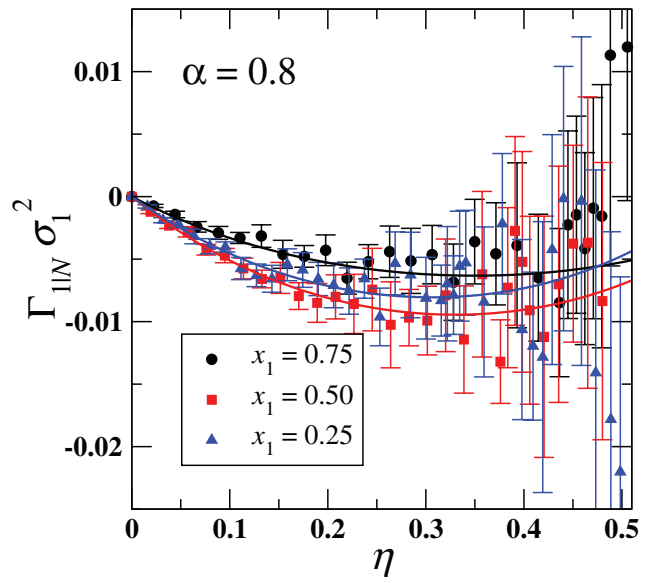

FIG. 6. Same as Fig. 5, except for $\alpha=0.8$.

the $\Gamma_{1 \mid N}$ data does not significantly affect the precision of the calculation of $\gamma$. At low packing fractions where the simulated $\Gamma_{1 \mid N}$ is well converged, there is good agreement with the WBII expressions.

The interfacial free energy $\gamma$ is then calculated from the data for $v_{N}$ and $\Gamma_{1 \mid N}$ by numerically integrating Eq. (16) using the trapezoid rule. As a result, the error in $\gamma$ includes contribution from the error in $\Gamma_{1 \mid N}$; despite this, high precision is preserved. Based on our analysis, the numerical error from the trapezoid rule is significantly smaller than the corresponding statistical error. The results for $\gamma$ are plotted in Figs. 8-10 and given in tabular form in the supplementary material. ${ }^{35}$ Also plotted in Figs. 8-10 are the predictions from the WBII bulk theory. The WBII predictions are in excellent agreement with the simulation data, except for overestimations at the highest packing fractions $(\eta>0.45)$. We have included an analysis of the separate $v_{N}$ and $\Gamma_{1 \mid N}$ contributions to $\gamma$ in the supplementary material. ${ }^{35}$

From the $\gamma$ versus $\eta$ data presented in Figs. 8-10, it is difficult to assess directly the dependence of $\gamma$ on mole fraction. To better illustrate the composition dependence of the interfacial free energy, we plot $\gamma$ as a function of composition $\left(x_{1}\right)$ in Fig. 11 for selected fixed values of $\eta$. The correspond-

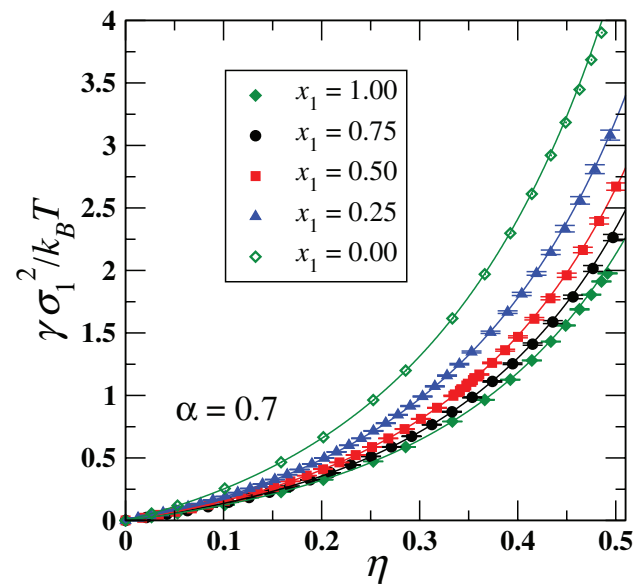

FIG. 8. Interfacial free energy per unit area, $\gamma$, as a function of packing fraction for the $\alpha=0.7$ mixtures. Results for the pure system from Laird and Davidchack ${ }^{10}$ are included (diamond symbols). Curves indicate $\gamma$ WBII. The inset is included to show better resolution at the higher pressures.

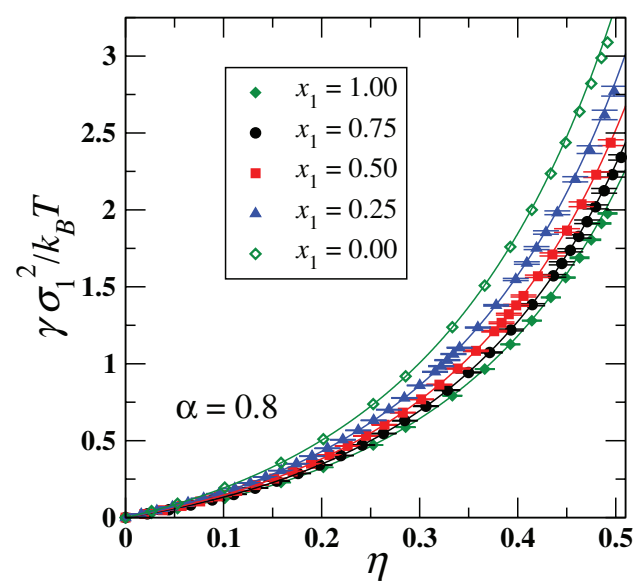

FIG. 9. Same as Fig. 8, except for $\alpha=0.8$ mixtures.

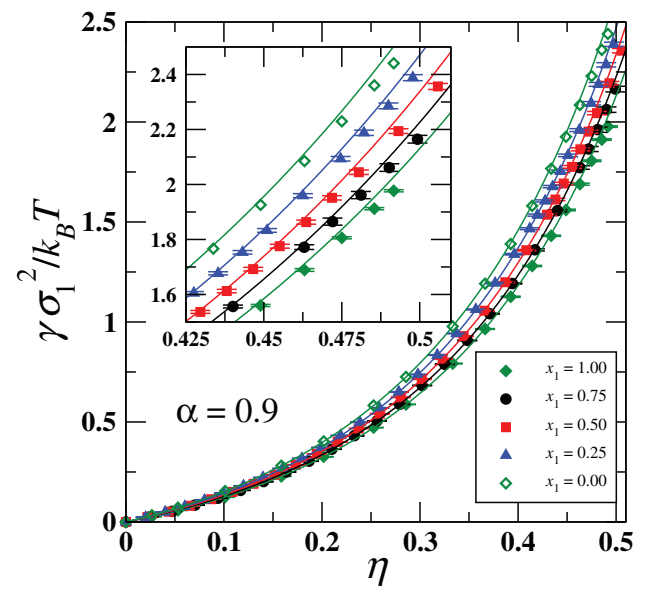

FIG. 10. Same as Fig. 8, except for $\alpha=0.9$ mixtures. 


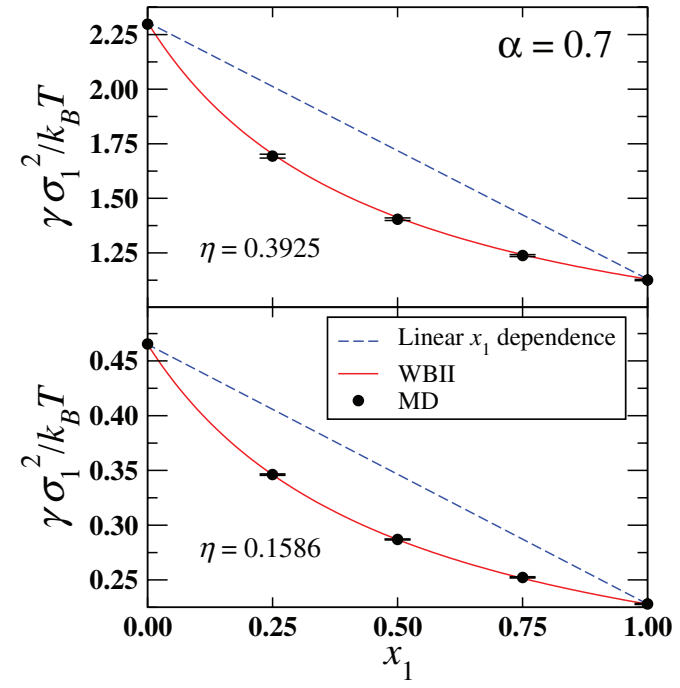

FIG. 11. Interfacial free energy per unit area, $\gamma$, as a function of $x_{1}$ for $\alpha=0.7$ mixtures. Values at these approximate $\eta$ were obtained by interpolation.

ing plots for $\alpha=0.8$ and 0.9 are shown in Figs. 12 and 13, respectively. The data for Figs. 11-13 were generated from the $\gamma$ versus $\eta$ data using quadratic interpolation. The simulation results show a significant negative deviation from linear dependence, which is well fit by the corresponding WBII results (also shown). The White Bear II theory predicts deviation from strict linear dependence, and we observe such deviation to a slightly greater extent than predicted. This deviation increases with increasing $\eta$ and is maximum near $x_{1}$ $=0.50$. As the $\Gamma_{1 \mid N}$ contribution to $\gamma$ is small and (generally) positive, this negative deviation from linearity is largely due to contributions from the excess volume, $v_{N}$.

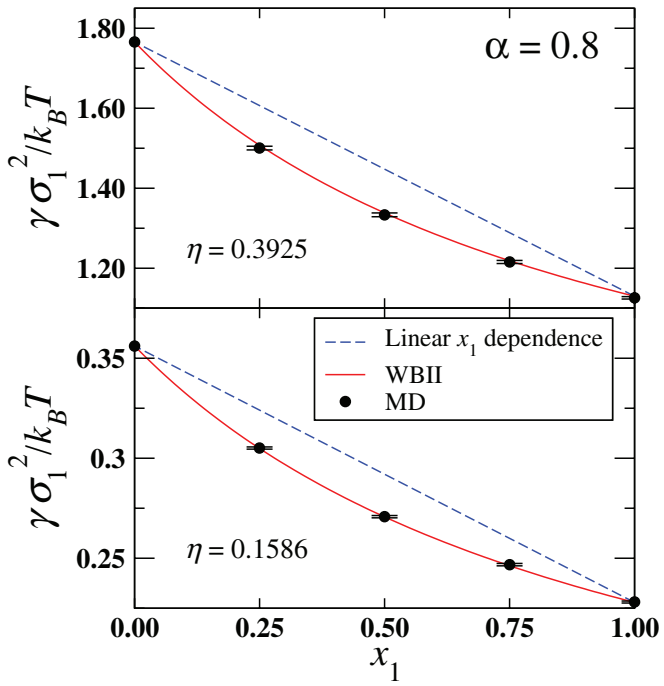

FIG. 12. Interfacial free energy per unit area, $\gamma$, as a function of $x_{1}$ for $\alpha=0.8$ mixtures. Values at these approximate $\eta$ were obtained by interpolation.

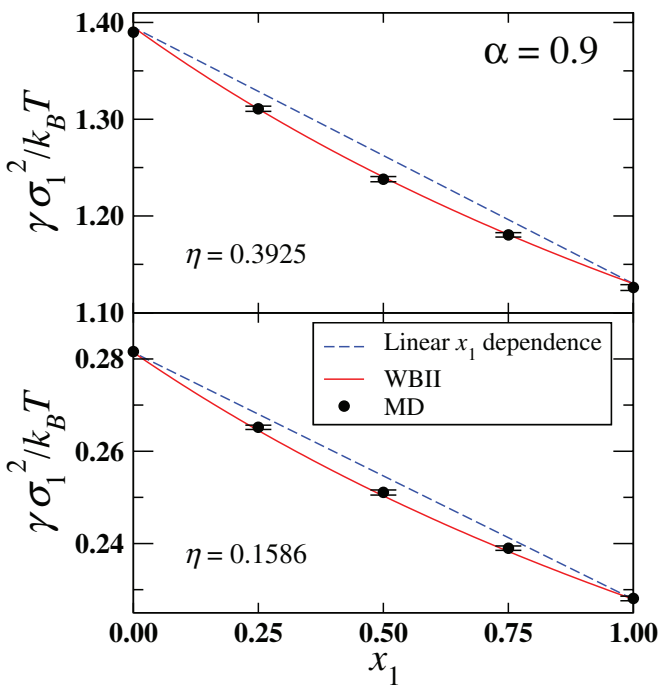

FIG. 13. Interfacial free energy per unit area, $\gamma$, as a function of $x_{1}$ for $\alpha=0.9$ mixtures. Values at these approximate $\eta$ were obtained by interpolation.

\section{SUMMARY}

In this work we use molecular-dynamics simulation to determine the interfacial thermodynamics of hard-sphere fluid mixtures at a hard wall as functions of density (or packing fraction) and composition $\left(x_{1}\right)$ for diameter ratios $\alpha=0.7$, 0.8 , and 0.9. The interfacial free energies, $\gamma$, for the systems studied are determined by first determining the excess interfacial volume $v_{N}$ and adsorption $\Gamma_{1 \mid N}$ for each $\alpha$, composition $\left(x_{1}\right)$ and packing fraction studied and then obtaining $\gamma$ by numerically integrating a Gibbs-Cahn adsorption equation. Comparison with the predictions from the WBII density functional ${ }^{14}$ shows that the WBII theory is in excellent agreement with the simulation data, except for small deviations at the highest packing fractions $(\eta>0.45)$. The results show that the contribution to $\gamma$ from the adsorption $\Gamma_{1 \mid N}$ is very small compared to that from the excess volume $\left(v_{N}\right)$ for the diameter ratios studied. For this reason, we are able to get high precision values of $\gamma$ at all packing fractions and compositions despite the large statistical errors in the measured values of the adsorption $\Gamma_{1 \mid N}$. Further investigation into mixtures with smaller diameter ratios at a hard wall would complement this work and others (for example, $\alpha=0.414$ ). ${ }^{36,37}$

\section{ACKNOWLEDGMENTS}

We gratefully acknowledge Professor Ruslan L. Davidchack and Professor Roland Roth for helpful discussions. We also would like to thank the National Science Foundation (NSF) for support under Grant No. CHE-0957102.

${ }^{1}$ V. D. Nguyen, Z. Hu, and P. Schall, Phys. Rev. E 84, 011607 (2011).

${ }^{2}$ H. Reiss, H. L. Frisch, E. Helfand, and J. L. Lebowitz, J. Chem. Phys. 32, 119 (1960).

${ }^{3}$ E. Helfand, J. L. Lebowitz, and E. Praestgaard, J. Chem. Phys. 43, 774-779 (1965).

${ }^{4}$ J. R. Henderson and F. van Swol, Mol. Phys. 51, 991 (1984).

${ }^{5}$ M. Heni and H. Löwen, Phys. Rev. E 60, 7057-7065 (1999). 
${ }^{6}$ A. Fortini and M. Dijkstra, J. Phys.: Condens. Matter 18, L371-L378 (2006).

${ }^{7}$ D. Deb, D. Wilms, A. Winkler, P. Virnau, and K. Binder, Int. J. Mod. Phys. C 23, 1240011 (2012).

${ }^{8}$ E. De Miguel and G. Jackson, Mol. Phys. 104, 3717-3734 (2006).

${ }^{9}$ B. B. Laird and R. L. Davidchack, J. Phys. Chem. C 111, 15952-15956 (2007).

${ }^{10}$ B. B. Laird and R. L. Davidchack, J. Chem. Phys. 132, 204101 (2010).

${ }^{11}$ B. B. Laird, A. Hunter, and R. L. Davidchack, Phys. Rev. E 86, 060602 (2012).

${ }^{12}$ Y. Rosenfeld, Phys. Rev. Lett. 63, 980-983 (1989).

${ }^{13}$ R. Roth, R. Evans, A. Lang, and G. Kahl, J. Stat. Phys.: Condens. Matter 14, 12063 (2002).

${ }^{14}$ H. Hansen-Goos and R. Roth, J. Stat. Phys.: Condens. Matter 18, 8413 (2006).

${ }^{15}$ A. R. Denton and N. W. Ashcroft, Phys. Rev. A 44, 8242-8248 (1991).

${ }^{16}$ R. Roth and S. Dietrich, Phys. Rev. E 62, 6926-6936 (2000).

${ }^{17}$ R. Roth, J. Phys.: Condens. Matter 22, 063102 (2010).

${ }^{18}$ J. P. Noworyta, D. Henderson, and S. Sokolowski, Mol. Phys. 95, 415 (1998).

${ }^{19}$ Z. Tan, U. M. B. Marconi, F. van Swol, and K. E. Gubbins, J. Chem. Phys. 90, 3704-3712 (1989).

${ }^{20}$ T. Frolov and Y. Mishin, Phys. Rev. B 79, 045430 (2009).

${ }^{21}$ T. Frolov and Y. Mishin, J. Chem. Phys. 131, 054702 (2009).

${ }^{22}$ B. B. Laird, R. L. Davidchack, Y. Yang, and M. Asta, J. Chem. Phys. 131, 114110 (2009).
${ }^{23}$ J. W. Cahn, "Thermodynamics of solid and fluid surfaces," in Interfacial Segregation, edited by W. C. Johnson and J. M. Blakely (ASM International, International Materials Park, OH, 1979), pp. 3-23.

${ }^{24}$ J. W. Gibbs, The Collected Works (Yale University Press, New Haven, 1957), Vol. 1, pp. 2887-2888.

${ }^{25}$ B. B. Laird, J. Chem. Phys. 115, 2888-2889 (2001).

${ }^{26} \mathrm{~W}$. A. Tiller, The Science of Crystallization: Microscopic Interfacial Phenomena (Cambridge University Press, New York, 1991).

${ }^{27}$ D. C. Rapaport, The Art of Molecular Dynamics Simulation, 2nd ed. (Cambridge University Press, New York, 2004).

${ }^{28}$ W. G. T. Kranendonk and D. Frenkel, Mol. Phys. 72, 679-697 (1991).

${ }^{29}$ X. Cottin and P. A. Monson, J. Chem. Phys. 107, 6855-6858 (1997).

${ }^{30}$ S. Punnathanam and P. A. Monson, J. Chem. Phys. 125, 24508-1-24508-8 (2006).

${ }^{31}$ B. Widom, J. Chem. Phys. 39, 2808-2812 (1963).

${ }^{32}$ P. Sindzingre, G. Ciccotti, C. Massobrio, and D. Frenkel, Chem. Phys. Lett. 136, 35-41 (1987).

${ }^{33}$ D. M. Heyes, Chem. Phys. 159, 149-167 (1992).

${ }^{34}$ G. A. Mansoori, N. F. Carnahan, K. E. Starling, and T. W. Leland, Jr., J. Chem. Phys. 54, 1523-1525 (1971).

${ }^{35}$ See supplementary material at http://dx.doi.org/10.1063/1.4858433 for tabulated data, as well as additional plots for pressure, chemical potential difference, and adsorption. Additional information as to the application of the White Bear Mark II density functional theory to this system is also included.

${ }^{36}$ R. Sibug-Aga and B. B. Laird, J. Chem. Phys. 116, 3410-3419 (2002).

${ }^{37}$ R. Sibug-Aga and B. B. Laird, Phys. Rev. B 66, 144106 (2002). 